\title{
Ecological niche modeling and new distribution records of the central dwarf frog $P$. centralis Bokermann, 1962 (Anura, Leptodactylidae, Leiuperinae) with comments on its taxonomic status
}

Daniel Loebmann, João Gabriel Ribeiro Giovanelli, Ana Cecília Giacometti Mai, Mariana Lúcio Lyra, Cinthia Aguirre Brasileiro \& Célio Fernando Baptista Haddad

To cite this article: Daniel Loebmann, João Gabriel Ribeiro Giovanelli, Ana Cecília Giacometti Mai, Mariana Lúcio Lyra, Cinthia Aguirre Brasileiro \& Célio Fernando Baptista Haddad (2017) Ecological niche modeling and new distribution records of the central dwarf frog $P$. centralis Bokermann, 1962 (Anura, Leptodactylidae, Leiuperinae) with comments on its taxonomic status, Tropical Zoology, 30:2, 49-67, DOI: 10.1080/03946975.2017.1278661

To link to this article: https://doi.org/10.1080/03946975.2017.1278661

Published online: 17 Feb 2017.

Submit your article to this journal ¿

LII Article views: 106

View Crossmark data 


\section{Ecological niche modeling and new distribution records of the central dwarf frog Physalaemus centralis Bokermann, 1962 (Anura, Leptodactylidae, Leiuperinae) with comments on its taxonomic status}

Daniel Loebmann $^{\mathrm{a}}$ (D)*, João Gabriel Ribeiro Giovanellib (iD, Ana Cecília Giacometti Mai ${ }^{\mathrm{a}}$, Mariana Lúcio Lyra ${ }^{\mathrm{b}}$ (D), Cinthia Aguirre Brasileiro ${ }^{\mathrm{C}}$ (D) and Célio Fernando Baptista Haddad ${ }^{\mathrm{b}}$

${ }^{a}$ Laboratório de Vertebrados. Av. Itália, Instituto de Ciências Biológicas, Universidade Federal do Rio Grande, km 8, Vila Carreiros, CEP 96.203-900, Rio Grande, RS, Brasil; 'baboratório de Herpetologia, Departamento de Zoologia, Instituto de Biociências, Universidade Estadual Paulista (UNESP), Av 24 A, 1515, Bela Vista, Caixa Postal 199, CEP 13506-970, Rio Claro, São Paulo, Brasil;; ${ }^{\circ}$ Laboratório de Ecologia e Sistemática, Departamento de Ciências Biológicas, Instituto de Ciências Ambientais, Químicas e Farmacêuticas, Universidade Federal de São Paulo, Rua Prof. Artur Riedel, 275, Eldorado, CEP: 09.972-270, Diadema, SP, Brasil

(Received 03 July 2015; accepted 04 October 2016; first published online 17 February 2017)

Physalaemus centralis is distributed over open areas of eastern South America. Herein, we perform an extensive review of occurrence records and propose a potential distribution based on ecological niche modeling. In order to confirm species identification, as well taxonomic status along its distribution, we provide morphological and molecular data from analysed specimens. To reach our goals, we compiled data available in the literature and from scientific collections, as well as unpublished data. We identified records of $P$. centralis in 123 localities, 86 of those records remain unpublished. Consequently, it is possible to extend its range of distribution to the state of Rondônia, Brazil, and also to the Caatinga formation. The Maxent algorithm provided a model of potential distribution consistent with the distribution proposed here. Results indicate that $P$. centralis has a large distribution associated to open areas of South America (Cerrado, Caatinga and Pantanal), with no evidence that this is a case of species complex. The multiple approach proposed herein, that combines occurrence data from different sources and molecular data to confirm species identification, provided consistent results. Therefore, we recommend that this framework be used as a protocol to assess species distribution in future studies, especially in the case of widely distributed species.

Keywords: Cerrado domain; distribution; modeling; Physalaemus cuvieri group; species complex

\section{Introduction}

Brazil shelters the richest amphibian fauna worldwide and over 1000 species have been regarded as valid taxa in the country (Frost 2016), which bears around $15 \%$ of the amphibian diversity recorded worldwide so far. Several species have been described in the past years (e.g. Brcko et al. 2013; Magalhães et al. 2014; Peloso et al. 2014). Nonetheless, the number of undescribed species remains high (see Loebmann and Haddad 2010; Fouquet et al. 2012). Keeping record of this surprisingly great amphibian biodiversity while their natural environments suffer with rapid destruction has become a great challenge for taxonomists, who have gathered efforts to discover and describe new species before they disappear. Additionally, the recognition of morphologically cryptic species has pushed

*Corresponding author: contato@danielloebmann.com 
taxonomy to a more complex level, demanding other sources of evidence beyond morphology. In this sense, molecular techniques have been broadly used to solve taxonomic confusion in many animal groups, including amphibians (e.g. Coloma et al. 2012; Teixeira et al. 2012).

Considering that amphibian biodiversity is not yet completely understood, one could expect that current knowledge about the geographic distribution of many amphibians is also greatly deficient (Vrcibradic et al. 2008), especially in countries with great biodiversity, such as Brazil. In fact, the subject remains so poorly understood that some scientific journals have maintained special sections to address the issue (see Check List, Herpetological Review, and Herpetology Notes).

Geographic distribution maps are often produced through expert-driven criteria (Giovanelli et al. 2010). These maps, also known as "range maps", are created using locations points from currently known distributions of the target species and aim to provide the limits of distribution (IUCN 2016). In amphibians, the Global Amphibian Assessment range maps are useful to showcase the known distribution of most species. However, points of occurrence of amphibians have been found far from the edges of such range maps in South America (Ficetola et al. 2014).

The use of statistical modeling methods to produce predictive distribution maps from species occurrence and environmental data (Guisan and Thuiller 2005) has increased considerably over the past years. These models are applied to meet several distinct scientific interests, including predicting potential invasion by alien species (e.g. Peterson et al. 2003; Papes and Peterson 2003; Ficetola et al. 2007; Giovanelli, Haddad, et al. 2008), assessing potential geographic distribution of species (e.g. Guisan and Thuiller 2005; Elith et al. 2006; Giovanelli, Araújo, et al. 2008), indicating geographical distribution of species in the past (e.g. Hugall et al. 2002) and providing additional information to plan conservation strategies and select protection areas (e.g. Jeganathan et al. 2004; Young 2007), among other interests.

The neotropical frog genus Physalaemus Fitzinger, 1826 is distributed from southern Mexico to northern Argentina (Nascimento et al. 2005), containing 47 recognized species (see Frost 2016). Most species in the Physalaemus cuvieri group (sensu Lourenço et al. 2015) are widely distributed, except for $P$. cuqui, that has a restricted distribution in northern Argentina and southern Bolivia (Lobo 1993; De La Riva et al. 2000), and P. erikae, which occurs only in Atlantic rainforest areas from southern Bahia (Cruz and Pimenta 2004). Four species from this group are distributed in the "dry diagonal" of the neotropics (Werneck 2011): Physalaemus albifrons and P. kroyeri are both associated to the Brazilian xeric formation called Caatinga, and $P$. albonotatus and $P$. centralis occur in open formations of Cerrado, Pantanal and Chaco ((see distribution details for these species in species in Frost 2016). Physalaemus cuvieri is the species with the largest distribution, occurring both in open areas such forest formations in eastern South America, although it has been recognized as a species complex (Lourenço et al. 2015). Herein, we provide information on the taxonomic status of $P$. centralis based on morphological and molecular data, and present an updated distribution map, including a distribution based on ecological niche modeling. The study was motivated by fact that this species' identification in northern Brazil is unclear, considering that $P$. centralis is morphologically similar to other species from the Physalaemus cuvieri group. Therefore, it is possible that $P$. centralis could be a species complex throughout its distribution. 


\section{Material and methods}

\section{Species identification}

To confirm identification of $P$. centralis throughout its distribution, we examined and compared specimens from 21 localities based on external morphological characters. All characteristics matched the original description (Bokermann 1962). Snout-vent length (SVL) was measured in other specimens from the Physalaemus cuvieri group once this parameter is useful to distinguish some species in the group. All analysed specimens were preserved and housed in the Célio F. B. Haddad amphibian collection (CFBH) located at UNESP (Departamento de Zoologia, Universidade Estadual Paulista, Rio Claro, São Paulo, Brazil), in a museum at UNICAMP (Museu de Zoologia Adão José Cardoso: ZUEC, Universidade Estadual de Campinas, Campinas, São Paulo, Brazil) and in a museum at USP (Museu de Zoologia da Universidade de São Paulo: MUZUSP, São Paulo, Brazil) (Appendix 1).

Additionally, we obtained partial sequences of the cytochrome-c oxidase subunit 1 (COI) gene and the $16 \mathrm{~S}$ ribosomal RNA (16S) gene from specimens of $P$. centralis collected at 12 localities of six Brazilian states (Appendix 2). In order to quantify inter-species divergence, we included sequences available in GenBank for P. centralis and out-group sequences of other closely related Physalaemus species in all analyses of COI and $16 \mathrm{~S}$ (Appendix 2).

Genomic DNA was extracted from ethanol-preserved muscle from each individual using QIAquick DNEasy kits (Qiagen, Hilden, Germany), following the manufacturer's protocol. Fragments were obtained via PCR using primers and conditions described in Lyra et al. (2016). PCR products were submitted to enzymatic purification and sequenced on an automated DNA sequencer (Applied Biosystems ABI 3130XL) at Macrogen Inc. (Seoul, South Korea) and at the Southern China DNA Barcode Center, Kunming Institute of Zoology, Chinese Academy of Science. GenBank accession numbers for each gene fragment are listed in Appendix 2. Gene sequences and metadata were also deposited at the Barcode of Life Data Systems (BOLD; Ratnasingham and Hebert 2007) under project code "PCENT".

Sequences were visualized and assembled into contigs using Geneious v6.0 (Biomatters). Sequence alignment was performed with Muscle (Edgar 2004) and verified by eye. Sequence distances were quantified using the uncorrected p-distance and complete deletion of gaps and missing data, and displayed in a neighbour-joining (NJ) tree, using MEGA6 software (Tamura et al. 2013). Node supports were assessed using 1000 bootstrap replicates.

\section{Data from species records and ecological niche modeling development}

In order to provide an updated distribution map for $P$. centralis, we compiled data available in the literature and in Brazilian scientific collections, in addition to new records of occurrence presented in this work. Localities of occurrence were obtained from the following scientific collections: CFBH; Coleção de Herpetologia, Universidade de Brasília (CHUNB), Brasília, Brazil; and Coleção de Vertebrados, Universidade Federal do Mato Grosso (UFMT), Cuiabá, Brazil. In addition, we accessed the online database of the species's Link project (2014) and obtained additional records of $P$. centralis from a collection at UNESP (Coleção do Departamento de Zoologia de São José do Rio Preto (DZSJRP), Universidade Estadual Paulista, São José do Rio Preto, Brazil) and from a collection at UNICAMP (Coleção de Anfíbios do Museu de História Natural "Prof. Dr. Adão José Cardoso" (ZUEC-AMP), Universidade Estadual de Campinas, Campinas, Brazil). 
Table 1. Mean and standard deviation of snout-vent length for species of Physalaemus cuvieri group.

\begin{tabular}{|c|c|c|c|}
\hline Species & Males & Females & Source \\
\hline Physalaemus albifrons & $28.6 \pm 2.0(N=7)$ & $29.7 \pm 4.9(N=41)$ & $\begin{array}{l}\text { Loebmann (unpub. } \\
\text { data) }\end{array}$ \\
\hline $\begin{array}{l}\text { Physalaemus albono- } \\
\text { tatus }\end{array}$ & $24.9 \pm 1.9(N=4)$ & $23.5 \pm 2.3(N=41)$ & $\begin{array}{l}\text { Brasileiro and Haddad } \\
\text { (2015) and Prado and } \\
\text { Haddad (2005) }\end{array}$ \\
\hline Physalaemus centralis & $36.3 \pm 2.4(N=27)$ & $34.5 \pm 2.7(N=9)$ & $\begin{array}{l}\text { Brasileiro and Martins } \\
\text { (2006) }\end{array}$ \\
\hline Physalaemus cuqui & $30.0 \pm 1.2(N=25)$ & & Lobo (1993) \\
\hline Physalaemus cuvieri & $27.6 \pm 3.0(N=525)$ & $29.5 \pm 2.7(N=680)$ & Brasileiro et al. (2005) \\
\hline Physalaemus ephippifer & $24.7 \pm 1.0(N=6)$ & $27.5 \pm 2.1(N=2)$ & $\begin{array}{l}\text { Brasileiro and Haddad } \\
\text { (2015) }\end{array}$ \\
\hline Physalaemus erikae & $23.7 \pm 2.1(N=5)$ & $23.1 \pm 2.3(N=13)$ & $\begin{array}{l}\text { Cruz and Pimenta } \\
\text { (2004) }\end{array}$ \\
\hline Physalaemus fischeri & $25.5 \pm 2.4(N=5)$ & & $\begin{array}{l}\text { Brasileiro and Haddad } \\
\text { (2015) }\end{array}$ \\
\hline Physalaemus kroyeri & $26.3 \pm 2.3(N=3)$ & & $\begin{array}{l}\text { Brasileiro and Haddad } \\
\text { (2015) }\end{array}$ \\
\hline
\end{tabular}

Species records were used to perform the ecological niche modeling. To check data consistency for the geographical positioning and to construct a map of species distribution, all records of occurrence were computed to DIVA-GIS software, version 7.5.0.0. Ecological niche modeling was performed using Maxent, version 3.3.3k (Phillips et al. 2006, available at http://www.cs.princeton.edu/ schapire/maxent/). The Maxent algorithm has been ranked among the most effective methods for species distribution modeling using presence-only data (Elith et al. 2006; Giovanelli et al. 2010). To run the model, we used 19 bioclimatic interpolated climate surfaces for global land areas (1950-2000) and a Derived Hydrological Digital Elevation Model (DEM-H). This data set is based on weather conditions recorded between 1950 and 2000, with 2.5 min resolution (Hijmans et al. 2005). The resulting model was evaluated with the receiver operating characteristics curve, calculating the area under the curve (AUC) (Fielding and Bell 1997).

We used the minimum presence threshold that equals the minimum model prediction value for any of the training occurrence point data, thus avoiding inflating commission errors (see Giovanelli et al. 2010). We generated a binary map depicting the predicted area for the model using a threshold value.

\section{Results and discussion}

\section{Species identification}

A total of 39 specimens of $P$. centralis were checked considering external morphology. All specimens were in accordance with the variation proposed in the original description (Bokermann 1962). One relevant aspect that is not mentioned in the species description refers to the colouration of the axillary and inguinal regions in live specimens, which are both orange in $P$. centralis. This is an important characteristic because it distinguishes $P$. centralis from other species of the $P$. cuvieri group. In P. cuvieri, this colouration is predominately reddish, while in $P$. albonotatus, $P$. cuqui and $P$. kroyeri, these regions are 
whitish (Lobo 1993). SVL comparisons among species show that $P$. centralis is larger than other species in the Physalaemus cuvieri group, reaching up to $43 \mathrm{~mm}$ of SVL (Bokermann 1962) (see SVL for other species of the group in Table 1).

Molecular analyses corroborate assumptions based on morphological data, showing that P. centralis has a large distribution associated to open areas of South America and there is no evidence that this species is a species complex throughout its geographical distribution. Mean intraspecific genetic distances found for COI and 16S were 0.01 (range 0.0-0.034) and 0.019 (range 0.0-0.027), respectively. These values are within the range expected for intraspecific distances in amphibians, in general less than $0.03-0.05$ for $16 \mathrm{~S}$ and 0.1 for COI (Fouquet et al. 2007). Distances between P. centralis and other Physalaemus species ranged from 0.118 (P. centralis vs. P. cuvieri) to 0.209 (P. centralis vs. P. nattereri) for COI and from 0.098 (P. centralis vs. P. cuvieri) to 0.175 (P. centralis vs. P. nattereri) for 16S. Although Jansen et al. (2011) suggested the existence of cryptic species in $P$. centralis from Bolivia based on molecular data ( 0.034 of divergence), our results suggest that it is a case of intraspecific variation with a weak geographic structure (see Figure 1).

\section{Distribution records and ecological niche modeling of Physalaemus centralis}

Compiled data provided 123 georeferenced records for $P$. centralis, 37 recovered from the literature, 74 coming from scientific collections and 12 being new occurrence records (Table 2). Those records are distributed between the countries of Bolivia (1 record), Brazil (115 records) and Paraguay (4 records). The edges of the geographic distribution are marked by coordinates $63.7097^{\circ}-41.3920^{\circ} \mathrm{W}$ and $2.4878^{\circ}-23.8156^{\circ} \mathrm{S}$ and altitude ranges from sea level to $1265 \mathrm{~m}$ above sea level (Figure 2). Our findings include the first record for the state of Rondônia (CFBH 20424), extending the species' distribution in Brazil in about $490 \mathrm{~km}$ in a straight-line westward of the Lucas do Rio Verde municipality, in the state of Mato Grosso (UFMT 2454, 2462, 2476, 2482, 2485, 2487). In addition, our records for the states of Bahia and Piauí are the first to mark this specie's occurrence in the Caatinga.

In Brazil, P. centralis was previously reported in the states of Bahia, Goiás, Maranhão, Mato Grosso, Mato Grosso do Sul, Minas Gerais, Piauí, São Paulo and Tocantins (Bokermann 1962; Strüssmann et al. 2000; Toledo et al. 2003; Kokubum and Menin 2002; Gordo and Campos 2003; Nascimento et al. 2005; Vasconcelos and Rossa-Feres 2005; Brasileiro and Martins 2006; Silveira 2006; Van-Silva et al. 2007; Brasileiro et al. 2008; Valdujo et al. 2009; Roberto et al. 2013). Data compilation shows that $P$. centralis is widely distributed in eastern South America, especially in open areas of Cerrado. However, we now show that the species also occurs in contact areas between the Cerrado and forested areas from closed formations of the Atlantic and Amazon rainforests. Admittedly, records from the Atlantic and Amazon forests are strongly associated with disturbed areas, i.e. areas deforested by anthropic activities. These results indicate that $P$. centralis is an opportunistic species and may extend its range of distribution with the advancement of deforestation. On the other hand, the species does not seem to tolerate extreme climatic conditions regarding temperature and precipitation in the Caatinga, keeping in mind that records presented here are limited to ecotonal areas of Cerrado and Caatinga.

The Maxent modeling of 123 presence records yielded an AUC of 0.94. Figure 3 shows the results of the jackknife test of variable importance generated by Maxent. The environmental variable with the highest gain when used in isolation is Temperature Annual Range (BIO7). The environmental variable that decreases in gain the most when omitted is Annual Precipitation (BIO12), which therefore appears to provide more information compared to other variables. 

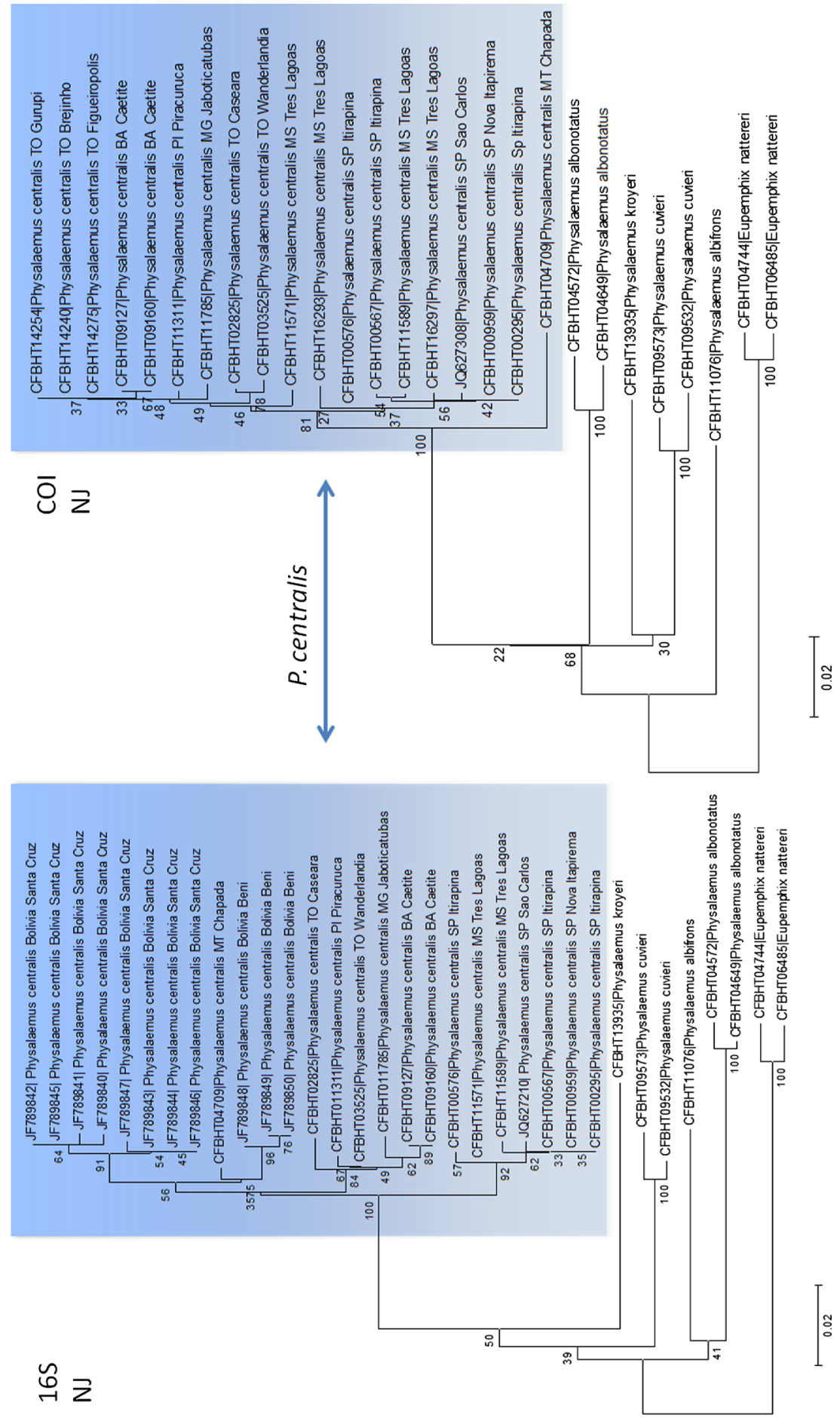

Figure 1. Neighbour-joining trees for $16 \mathrm{~S}$ and COI of analysed Physalaemus species. All samples of Physalaemus centralis are grouped into a single clade. Out-groups are represented by other Physalaemus species. 
Table 2. Records of Physalaemus centralis in Brazil based on field work (present study), bibliographical data, and voucher specimens in Brazilian collections.

\begin{tabular}{|c|c|c|c|c|c|}
\hline Municipality & State/Province & Country & Longitude & Latitude & Source \\
\hline Capitán Bado & Amambay & Paraguay & -55.5554 & -23.2661 & $\begin{array}{l}\text { Brusquetti and } \\
\text { Lavilla (2006) }\end{array}$ \\
\hline $\begin{array}{l}\text { Pedro Juan Ca- } \\
\text { ballero }\end{array}$ & Amambay & Paraguay & -56.0546 & -22.6218 & $\begin{array}{l}\text { Brusquetti and } \\
\text { Lavilla (2006) }\end{array}$ \\
\hline $\begin{array}{l}\text { Santa Rosa del } \\
\text { Aguaray }\end{array}$ & San Pedro & Paraguay & -56.2952 & -23.8126 & $\begin{array}{l}\text { Smith et al. } \\
\text { (2012) }\end{array}$ \\
\hline Caetité & Bahia & Brazil & -42.4833 & -14.0667 & $\begin{array}{l}\text { Present study } \\
\text { (CFBH 21079, } \\
21080 \text { ) }\end{array}$ \\
\hline Cocos & Bahia & Brazil & -44.5344 & -14.1839 & CHUNB \\
\hline Correntina & Bahia & Brazil & -44.6367 & -13.3433 & CHUNB \\
\hline Jaborandi & Bahia & Brazil & -44.4328 & -13.6194 & CHUNB \\
\hline Lençóis & Bahia & Brazil & -41.3920 & -12.5574 & $\mathrm{CFBH}$ \\
\hline São Desidério & Bahia & Brazil & -45.0833 & -12.7000 & $\begin{array}{l}\text { Valdujo et al. } \\
\text { (2009) }\end{array}$ \\
\hline São Desidério & Bahia & Brazil & -44.6000 & -13.0167 & $\begin{array}{l}\text { Valdujo et al. } \\
\text { (2009) }\end{array}$ \\
\hline São Desidério & Bahia & Brazil & -44.9667 & -12.3667 & CHUNB \\
\hline Iténez & Beni & Bolivia & -63.7097 & -13.2648 & $\begin{array}{l}\text { Padial and } \\
\text { Köhler (2001) }\end{array}$ \\
\hline Itanará & Canindeyú & Paraguay & -55.7801 & -23.8156 & $\begin{array}{l}\text { Brusquetti and } \\
\text { Lavilla (2006) }\end{array}$ \\
\hline Acreúna & Goiás & Brazil & -50.3883 & -17.3867 & $\mathrm{CFBH}$ \\
\hline $\begin{array}{l}\text { Alvorada do } \\
\text { Norte }\end{array}$ & Goiás & Brazil & -46.4922 & -14.4808 & CHUNB \\
\hline Anápolis & Goiás & Brazil & -48.9257 & -16.3112 & $\mathrm{CFBH}$ \\
\hline Aporé & Goiás & Brazil & -51.8806 & -18.6739 & $\begin{array}{l}\text { Van-Silva et al. } \\
(2007)\end{array}$ \\
\hline Baliza & Goiás & Brazil & -52.545 & -16.1961 & $\mathrm{CFBH}$ \\
\hline Britânia & Goiás & Brazil & -51.1500 & -15.2333 & CHUNB \\
\hline Catalão & Goiás & Brazil & -47.9500 & -18.1667 & CHUNB \\
\hline $\begin{array}{l}\text { Corumbá de } \\
\text { Goiás }\end{array}$ & Goiás & Brazil & -48.8000 & -15.9167 & CHUNB \\
\hline Indiara & Goiás & Brazil & -49.9809 & -17.1273 & $\mathrm{CFBH}$ \\
\hline Mineiros & Goiás & Brazil & -52.5667 & -17.5667 & CHUNB \\
\hline $\begin{array}{l}\text { Palmeiras de } \\
\text { Goiás }\end{array}$ & Goiás & Brazil & -49.9384 & -16.7897 & $\mathrm{CFBH}$ \\
\hline Rio Verde & Goiás & Brazil & -50.9446 & -17.7718 & $\mathrm{CFBH}$ \\
\hline São Domingos & Goiás & Brazil & -46.3167 & -13.4000 & CHUNB \\
\hline São Simão & Goiás & Brazil & -50.5332 & -19.0056 & $\mathrm{CFBH}$ \\
\hline Silvânia & Goiás & Brazil & -48.6081 & -16.6589 & $\begin{array}{l}\text { Nascimento et al. } \\
\text { (2005) }\end{array}$ \\
\hline Carolina & Maranhão & Brazil & -47.4694 & -7.3328 & Present study \\
\hline
\end{tabular}


Table 2. (Continued).

\begin{tabular}{|c|c|c|c|c|c|}
\hline Municipality & State/Province & Country & Longitude & Latitude & Source \\
\hline Estreito & Maranhão & Brazil & -47.4286 & -6.0106 & $\begin{array}{l}\text { Brasileiro et al. } \\
(2008)\end{array}$ \\
\hline Estreito & Maranhão & Brazil & -47.3614 & -6.2733 & $\begin{array}{l}\text { Brasileiro et al. } \\
(2008)\end{array}$ \\
\hline Porto Franco & Maranhão & Brazil & -47.4000 & -6.3333 & $\begin{array}{l}\text { Present study } \\
\text { (CFBH 25953) }\end{array}$ \\
\hline $\begin{array}{l}\text { Santo Amaro do } \\
\text { Maranhão }\end{array}$ & Maranhão & Brazil & -43.2928 & -2.4878 & $\mathrm{CFBH}$ \\
\hline $\begin{array}{l}\text { Chapada dos Gui- } \\
\text { marães }\end{array}$ & Mato Grosso & Brazil & -55.7497 & -15.4606 & $\mathrm{CFBH}$ \\
\hline $\begin{array}{l}\text { Chapada dos Gui- } \\
\text { marães }\end{array}$ & Mato Grosso & Brazil & -55.7500 & -15.4333 & CHUNB \\
\hline Cuiabá & Mato Grosso & Brazil & -56.0833 & -15.5833 & CHUNB \\
\hline $\begin{array}{l}\text { Lucas do Rio } \\
\text { Verde }\end{array}$ & Mato Grosso & Brazil & -55.8899 & -13.1001 & UFMT \\
\hline $\begin{array}{l}\text { Nossa Senhora do } \\
\text { Livramento }\end{array}$ & Mato Grosso & Brazil & -53.3323 & -15.7882 & UFMT \\
\hline Paranatinga & Mato Grosso & Brazil & -54.0557 & -14.4723 & UFMT \\
\hline $\begin{array}{l}\text { Porto Alegre do } \\
\text { Norte }\end{array}$ & Mato Grosso & Brazil & -51.6325 & -10.8769 & CHUNB \\
\hline $\begin{array}{l}\text { Ribeirão Cascal- } \\
\text { heira }\end{array}$ & Mato Grosso & Brazil & -51.8241 & -12.9419 & ZUEC-AMP \\
\hline $\begin{array}{l}\text { São Félix do } \\
\text { Araguaia }\end{array}$ & Mato Grosso & Brazil & -50.6691 & -11.6172 & ZUEC-AMP \\
\hline Xingu & Mato Grosso & Brazil & -52.4031 & -12.0056 & $\begin{array}{l}\text { Nascimento et al. } \\
2005 \text {; }\end{array}$ \\
\hline Bonito & $\begin{array}{c}\text { Mato Grosso do } \\
\text { Sul }\end{array}$ & Brazil & -56.5056 & -20.6508 & $\begin{array}{l}\text { Gordo and } \\
\text { Campos (2003) }\end{array}$ \\
\hline Dourados & $\begin{array}{l}\text { Mato Grosso do } \\
\text { Sul }\end{array}$ & Brazil & -54.8056 & -22.2211 & $\begin{array}{l}\text { Tombini and } \\
\text { Béda (2002) }\end{array}$ \\
\hline Três Lagoas & $\begin{array}{l}\text { Mato Grosso do } \\
\text { Sul }\end{array}$ & Brazil & -51.7718 & -20.9762 & $\begin{array}{l}\text { Present study } \\
\text { (CFBH CFBH } \\
13595,18482, \\
18483,22631 \text {, } \\
24016,24020 \text {, } \\
24040)\end{array}$ \\
\hline Belo Horizonte & Minas Gerais & Brazil & -43.9378 & -19.9208 & $\mathrm{CFBH}$ \\
\hline Buritis & Minas Gerais & Brazil & -46.4233 & -15.6178 & $\begin{array}{l}\text { Nascimento et al. } \\
\text { (2005) }\end{array}$ \\
\hline Buritizeiro & Minas Gerais & Brazil & -44.9622 & -17.3511 & $\begin{array}{l}\text { Nascimento et al. } \\
\text { (2005) }\end{array}$ \\
\hline Chapada Gaúcha & Minas Gerais & Brazil & -45.6183 & -15.3056 & CHUNB \\
\hline Conquista & Minas Gerais & Brazil & -47.5417 & -19.9372 & $\begin{array}{l}\text { Nascimento et al. } \\
(2005)\end{array}$ \\
\hline Diamantina & Minas Gerais & Brazil & -43.6003 & -18.2494 & $\begin{array}{l}\text { Nascimento et al. } \\
(2005)\end{array}$ \\
\hline
\end{tabular}


Table 2. (Continued).

\begin{tabular}{|c|c|c|c|c|c|}
\hline Municipality & State/Province & Country & Longitude & Latitude & Source \\
\hline Esmeraldas & Minas Gerais & Brazil & -44.3138 & -19.7628 & UNICAMP \\
\hline Formoso & Minas Gerais & Brazil & -46.2319 & -14.9467 & CHUNB \\
\hline Jaboticatubas & Minas Gerais & Brazil & -43.7450 & -19.5136 & ZUEC-AMP \\
\hline João Pinheiro & Minas Gerais & Brazil & -46.1667 & -17.7333 & Silveira (2006) \\
\hline Lagoa Santa & Minas Gerais & Brazil & -43.8897 & -19.6272 & $\begin{array}{l}\text { Nascimento et al. } \\
(2005)\end{array}$ \\
\hline Manga & Minas Gerais & Brazil & -43.9322 & -14.7558 & $\begin{array}{l}\text { Nascimento et al. } \\
(2005)\end{array}$ \\
\hline Pirapora & Minas Gerais & Brazil & -44.9419 & -17.345 & $\begin{array}{l}\text { Nascimento et al. } \\
(2005)\end{array}$ \\
\hline Porteirinha & Minas Gerais & Brazil & -43.0283 & -15.7433 & $\begin{array}{l}\text { Nascimento et al. } \\
(2005)\end{array}$ \\
\hline $\begin{array}{l}\text { Riacho dos } \\
\text { Machados }\end{array}$ & Minas Gerais & Brazil & -43.0494 & -16.0061 & $\begin{array}{l}\text { Nascimento et al. } \\
(2005)\end{array}$ \\
\hline Sacramento & Minas Gerais & Brazil & -47.4400 & -19.8653 & $\begin{array}{l}\text { Nascimento et al. } \\
(2005)\end{array}$ \\
\hline $\begin{array}{l}\text { Santana do } \\
\text { Riacho }\end{array}$ & Minas Gerais & Brazil & -43.7144 & -19.1689 & $\begin{array}{l}\text { Nascimento et al. } \\
(2005)\end{array}$ \\
\hline Uberlandia & Minas Gerais & Brazil & -48.3000 & -18.9833 & $\begin{array}{l}\text { Kokubum and } \\
\text { Menin (2002) }\end{array}$ \\
\hline União de Minas & Minas Gerais & Brazil & -50.3416 & -19.5359 & $\mathrm{CFBH}$ \\
\hline Várzea da Palma & Minas Gerais & Brazil & -44.7308 & -17.5981 & $\begin{array}{l}\text { Nascimento et al. } \\
(2005)\end{array}$ \\
\hline Piracuruca & Piauí & Brazil & -41.6789 & -4.1933 & $\begin{array}{l}\text { Present study } \\
\text { (CFBH 19396, } \\
\text { 19397) }\end{array}$ \\
\hline $\begin{array}{l}\text { Colorado do } \\
\text { Oeste }\end{array}$ & Rondônia & Brazil & -60.3937 & -13.0385 & $\begin{array}{l}\text { Present study/ } \\
\text { New state record } \\
\text { (CFBH 20424) }\end{array}$ \\
\hline Angatuba & São Paulo & Brazil & -48.3844 & -23.5009 & $\mathrm{CFBH}$ \\
\hline Araraquara & São Paulo & Brazil & -48.1697 & -21.7903 & ZUEC-AMP \\
\hline Bauru & São Paulo & Brazil & -49.0600 & -22.3100 & DZSJRP \\
\hline Botucatu & São Paulo & Brazil & -48.445 & -22.8858 & $\begin{array}{l}\text { Nascimento et al. } \\
(2005)\end{array}$ \\
\hline Brotas & São Paulo & Brazil & -48.1267 & -22.2842 & $\mathrm{CFBH}$ \\
\hline Corumbataí & São Paulo & Brazil & -47.6258 & -22.2200 & $\begin{array}{l}\text { Nascimento et al. } \\
(2005)\end{array}$ \\
\hline Guaíra & São Paulo & Brazil & -48.3106 & -20.3183 & DZSJRP \\
\hline Ibirá & São Paulo & Brazil & -49.2408 & -21.0803 & DZSJRP \\
\hline Icem & São Paulo & Brazil & -49.195 & -20.3417 & UNESPSJRP \\
\hline Itirapina & São Paulo & Brazil & -47.8228 & -22.2528 & $\mathrm{CFBH}$ \\
\hline Jaguariuna & São Paulo & Brazil & -46.9797 & -22.7003 & ZUEC-AMP \\
\hline José Bonifácio & São Paulo & Brazil & -49.6883 & -21.0528 & UNESPSJRP \\
\hline
\end{tabular}


Table 2. (Continued).

\begin{tabular}{|c|c|c|c|c|c|}
\hline Municipality & State/Province & Country & Longitude & Latitude & Source \\
\hline Luiz Antônio & São Paulo & Brazil & -47.7987 & -21.6057 & $\begin{array}{l}\text { Prado et al. } \\
\text { (2009) }\end{array}$ \\
\hline Magda & São Paulo & Brazil & -50.2261 & -20.6439 & UNESPSJRP \\
\hline Nova Aliança & São Paulo & Brazil & -49.5431 & -21.0761 & UNESPSJRP \\
\hline Nova Granada & São Paulo & Brazil & -49.3142 & -20.5339 & DZSJRP \\
\hline Nova Itapirema & São Paulo & Brazil & -49.5333 & -21.0667 & $\begin{array}{l}\text { Vasconcelos } \\
\text { and Rossa-Feres } \\
(2005)\end{array}$ \\
\hline Novo Horizonte & São Paulo & Brazil & -49.2208 & -21.4681 & DZSJRP \\
\hline Palestina & São Paulo & Brazil & -49.4300 & -20.3900 & ZUEC-AMP \\
\hline Paulínia & São Paulo & Brazil & -47.1500 & -22.7603 & ZUEC-AMP \\
\hline Pirassununga & São Paulo & Brazil & -47.4244 & -21.9978 & $\begin{array}{l}\text { Brasileiro and } \\
\text { Martins (2006) }\end{array}$ \\
\hline Planalto & São Paulo & Brazil & -49.9739 & -21.0014 & FAPESP \\
\hline Rio Claro & São Paulo & Brazil & -47.5500 & -22.4167 & $\begin{array}{l}\text { Toledo et al. } \\
\text { (2003) }\end{array}$ \\
\hline Santa Fé do Sul & São Paulo & Brazil & -50.9258 & -20.2111 & UNESPSJRP \\
\hline $\begin{array}{l}\text { Santo Antônio do } \\
\text { Aracanguá }\end{array}$ & São Paulo & Brazil & -50.3490 & -20.9260 & DZSJRP \\
\hline $\begin{array}{l}\text { São José do Rio } \\
\text { Preto }\end{array}$ & São Paulo & Brazil & -49.3794 & -20.8197 & UNESPSJRP \\
\hline Tanabi & São Paulo & Brazil & -49.6492 & -20.6264 & UNESPSJRP \\
\hline Turmalina & São Paulo & Brazil & -50.4655 & -20.0521 & $\mathrm{CFBH}$ \\
\hline União Paulista & São Paulo & Brazil & -49.9261 & -20.9211 & FAPESP \\
\hline Vitoria Brasil & São Paulo & Brazil & -50.4844 & -20.1967 & UNESPSJRP \\
\hline Votuporanga & São Paulo & Brazil & -49.9728 & -20.4228 & UNESPSJRP \\
\hline Araguacema & Tocantins & Brazil & -49.5564 & -8.8036 & $\begin{array}{l}\text { Present study } \\
\text { (CFBH 10313) }\end{array}$ \\
\hline Araguaina & Tocantins & Brazil & -48.2072 & -7.1911 & Present study \\
\hline Babaçulândia & Tocantins & Brazil & -48.0153 & -7.0842 & $\begin{array}{l}\text { Brasileiro et al. } \\
(2008)\end{array}$ \\
\hline Cazeara & Tocantins & Brazil & -49.9556 & -9.2783 & $\mathrm{CFBH}$ \\
\hline $\begin{array}{l}\text { Colinas do To- } \\
\text { cantins }\end{array}$ & Tocantins & Brazil & -48.475 & -8.0592 & CHUNB \\
\hline Darcinópoles & Tocantins & Brazil & -47.7608 & -6.7147 & $\begin{array}{l}\text { Brasileiro et al. } \\
(2008)\end{array}$ \\
\hline Dois Irmãos & Tocantins & Brazil & -48.0878 & -6.7808 & Present study \\
\hline $\begin{array}{l}\text { Formoso do } \\
\text { Araguaia }\end{array}$ & Tocantins & Brazil & -49.5289 & -11.7967 & ZUEC-AMP \\
\hline Guaraí & Tocantins & Brazil & -48.5103 & -8.8342 & Present study \\
\hline Lizarda & Tocantins & Brazil & -46.6731 & -9.5942 & $\mathrm{CFBH}$ \\
\hline Mateiros & Tocantins & Brazil & -46.4211 & -10.5475 & CHUNB \\
\hline
\end{tabular}


Table 2. (Continued).

\begin{tabular}{lccccl}
\hline Municipality & State/Province & Country & Longitude & Latitude & Source \\
\hline Palmas & Tocantins & Brazil & -48.4361 & -10.4208 & CHUNB \\
Palmeirante & Tocantins & Brazil & -47.9258 & -7.8600 & Present study \\
Palmeirópolis & Tocantins & Brazil & -48.4022 & -13.0439 & CHUNB \\
Paraíso do To- & Tocantins & Brazil & -48.8667 & -10.1761 & CHUNB \\
cantins & & & & & \\
Paranã & Tocantins & Brazil & -47.8831 & -12.6153 & CHUNB \\
Pedro Afonso & Tocantins & Brazil & -48.1747 & -8.9675 & CHUNB \\
Porto Nacional & Tocantins & Brazil & -48.4172 & -10.7081 & CHUNB \\
São Félix & Tocantins & Brazil & -50.6703 & -11.5636 & Present study \\
Wanderlândia & Tocantins & Brazil & -47.9631 & -6.8492 & CFBH \\
\hline
\end{tabular}

Abbreviations: CFBH - Colecão de Anfíbios Célio F. B. Haddad; DZSJRP - Coleção do Departamento de Zoologia de São José do Rio Preto; ZUEC-AMP - Coleção de Anfíbios do Museu de História Natural "Prof. Dr. Adão José Cardoso"; CHUNB - Coleção Herpetológica da Universidade de Brasília; UFMT - Coleção de Vertebrados da Universidade Federal do Mato Grosso.

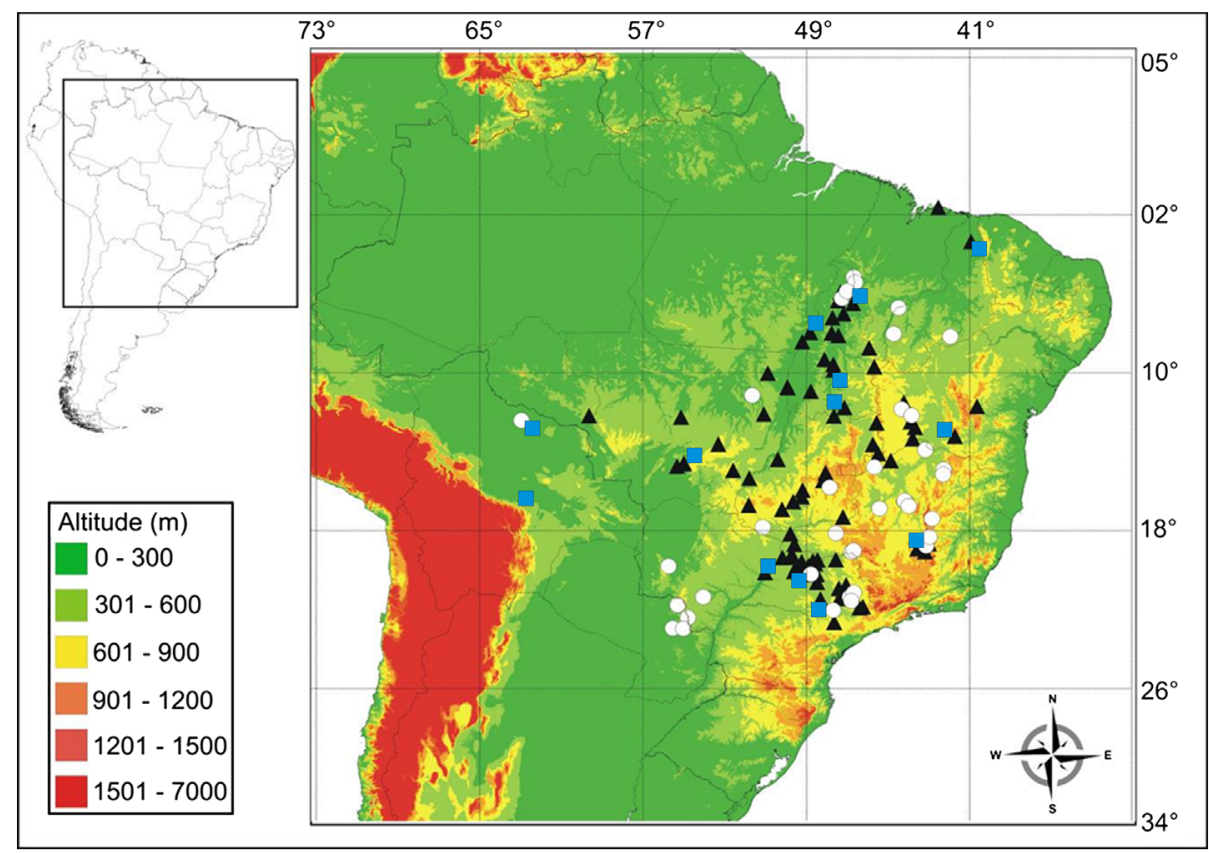

Figure 2. Map showing current known distribution of Physalaemus centralis. White circles represent records available in the literature. Black triangles are unpublished records (records obtained from scientific collections or presented for the first time in the present study). Blue squares represent specimens with molecular data accessed.

The ecological niche modeling suggests that $P$. centralis has potential to occur in open areas eastward of South America, including the Caatinga, Chaco (in Paraguay) and mainly the Cerrado (Figure 4). The model also points out that the Atlantic rainforest from south-eastern Brazil, as well as transitional zones between the Cerrado and the Amazon 


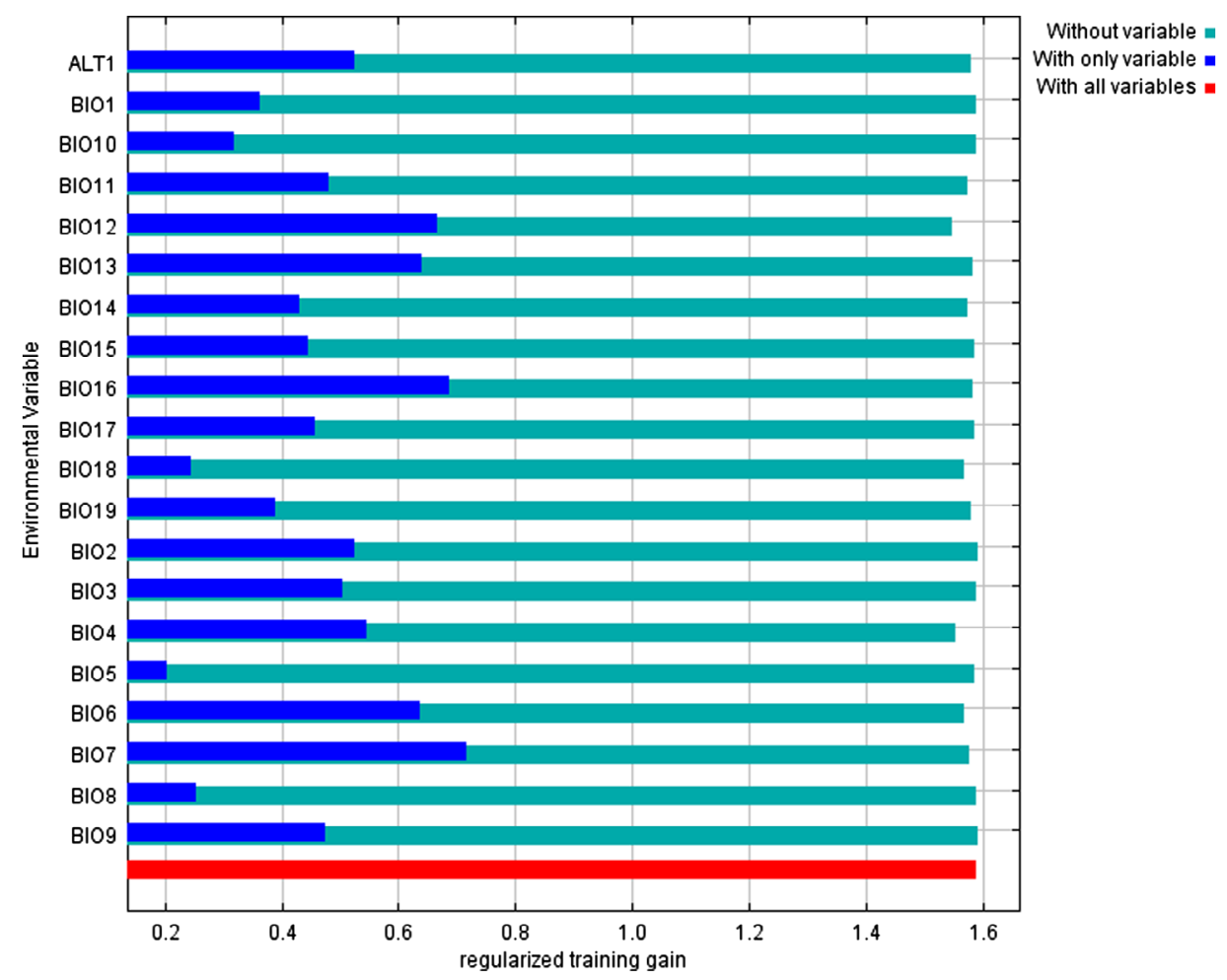

Figure 3. Results of the jackknife test of variable importance generating by Maxent. The environmental variables Temperature Annual Range (BIO7) and Annual Precipitation (BIO12) were the most important variables.

rainforest, are potential areas for species occurrence. It is possible that these regions have become suitable after the fragmentation of the Atlantic rainforest and the formation of the "arc of deforestation" in Amazonia, mainly in the states of Rondônia and Pará, which caused the species to invade recently opened areas.

However, some areas of occurrence predicted in the model should be discarded. For example, the occurrence prediction for the Plateau of Ibiapaba, in the state of Ceará, Brazil $\left(04^{\circ} \mathrm{S} ; 40^{\circ} \mathrm{W}\right)$ is probably a bias of the model, given that this region was exhaustively sampled in the years 2007-2009 and no P. centralis specimen was found (see Loebmann and Haddad 2010; Roberto and Loebmann 2016). The Plateau of Ibiapaba has a relatively high altitude (ca. $900 \mathrm{~m}$ above sea level) and extends throughout the frontier of the states of Piauí and Ceará. Although we have confirmed the presence of Physalaemus species in the state of Piauí, municipality of Piracuruca (ca. $40 \mathrm{~km}$ from the base of the Plateau of Ibiapaba), the high altitude from of the plateau may act as a barrier for the species. Therefore, the predicted occurrence of $P$. centralis in Ibiapaba, in other areas of the state of Ceará, as well as in the states of Pernambuco and Rio Grande do Norte, should be considered possible biases of the model. In conclusion, the model is relatively consistent with records of occurrence presented herein, with these few exceptions.

The present work used an inductive framework to develop maps of geographic distribution integrating occurrence data obtained from museums, a genetic approach 


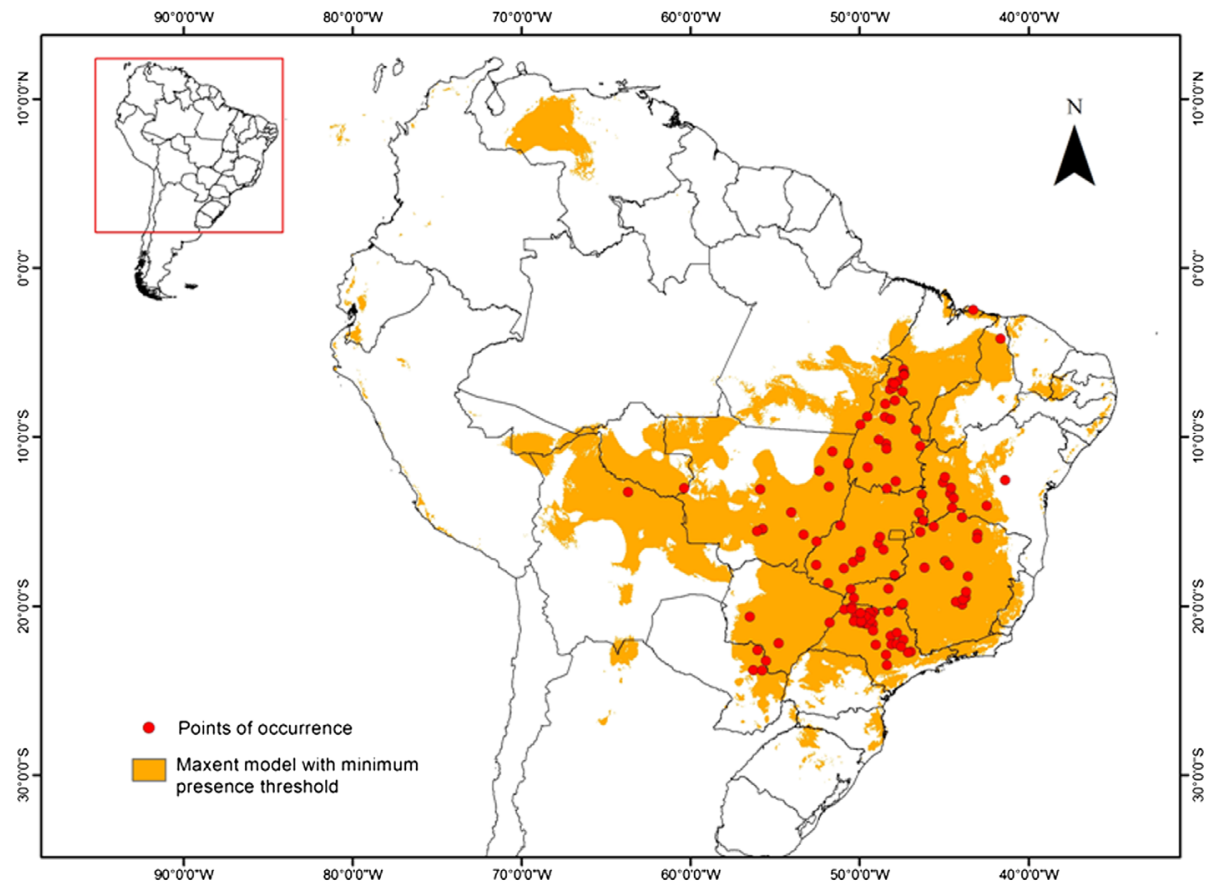

Figure 4. Prediction of the potential distribution of Physalaemus centralis based on the Maxent algorithm.

and ecological niche modeling. This multiple approach revealed that $P$. centralis is in fact widely distributed and strongly related to the Cerrado biome. We also conclude that $P$. centralis should not be considered a species complex. Therefore, we recommend that future studies involving widely distributed species should consider an integrative approach similar to what was developed here.

\section{Acknowledgements}

The authors are grateful to Dr. Guarino Colli (Curator of the CHUNB) and Dr. Marcos André de Carvalho (Curator from UFMT) for providing the data on Physalaemus centralis deposited in the collections of their responsibilities, to the Centro de Referência em Informação Ambiental (CRIA) for making the species records of several institutions available for non-profit proposes, which considerably improved the construction of the model, to the collections from the Museu de Zoologia of the Universidade Estadual de Campinas and the Coleção de Anfíbios from the Departamento de Zoologia de São José do Rio Preto that hosted their data for publications at CRIA, to the Centro de Estudos de Insetos Sociais (CEIS), UNESP, Rio Claro, Brazil and the Southern China DNA Barcode Center, Kunming Institute of Zoology, Chinese Academy of Science, for allowing us to use the facilities for molecular analyses.

\section{Disclosure statement}

No potential conflict of interest was reported by the authors. 


\section{Funding}

Daniel Loebmann [grant number 338632/2010] and João Gabriel Ribeiro Giovanelli were supported by the Coordenação de Aperfeiçoamento de Pessoal de Nível Superior (CAPES). Célio Haddad wishes to thank the Fundação de Amparo à Pesquisa do Estado de São Paulo (FAPESP) [grant \#2013/50741-7], and CAPES for a research fellowship from CNPq.

\section{ORCID}

Daniel Loebmann (D) http://orcid.org/0000-0003-0106-7197

João Gabriel Ribeiro Giovanelli (iD http://orcid.org/0000-0002-7314-9442

Mariana Lúcio Lyra (iD http://orcid.org/0000-0002-7863-4965

Cinthia Aguirre Brasileiro (D) http://orcid.org/0000-0001-8523-8621

\section{References}

Bokermann WCA. 1962. Sobre uma pequena coleção de anfíbios do Brasil Central, com de descrição de uma espécie nova de Physalaemus (Amphibia, Salientia). Revista Brasileira de Biologia. 22:213-219.

Brasileiro CA, Haddad CFB. 2015. A New Species of Physalaemus from Central Brazil (Anura: Leptodactylidae). Herpetologica. 71(4):280-288.

Brasileiro CA, Lucas EM, Oyamaguchi HM, Thomé MTC, Dixo M. 2008. Anurans, northern Tocantins River Basin, states of Tocantins and Maranhão, Brazil. Check List. 4:185-197.

Brasileiro CA, Martins M. 2006. Breeding biology of Physalaemus centralis Bokermann, 1962 (Anura: Leptodactylidae) in southeastern Brazil. Journal of Natural History. 40:1199-1209.

Brasileiro CA, Sawaya RJ, Kiefer MC, Martins M. 2005. Amphibian of an open Cerrado fragment in the southeastern Brazil. Biota Neotropica. 5(2):1-5.

Brcko I, Hoogmoed MS, Neckel-Oliveira S. 2013. Taxonomy and distribution of the salamander genus Bolitoglossa Duméril, Bibron and Duméril, 1854 (Amphibia, Caudata, Plethodontidae) in Brazilian Amazonia. Zootaxa. 3686:401-431.

Brusquetti F, Lavilla EO. 2006. Lista comentada de los anfibios de Paraguay. Cuadernos de Herpetología. 20(2):17.

Coloma LA, Carvajal-Endara S, Dueñas JF, Paredes-Recalde A, Morales-Mite M, Almeida-Reinoso D, Tapia EE, Hutter CR, Toral E, Guayasamin JM. 2012. Molecular phylogenetics of stream treefrogs of the Hyloscirtus larinopygion group (Anura: Hylidae), and description of two new species from Ecuador. Zootaxa. 3364:1-78.

Cruz CAG, Pimenta BVS. 2004. New species of Physalaemus Fitzinger, 1826 from southern Bahia, Brazil (Anura, Leptodactylidae). Journal of Herpetology. 38:480-486.

De La Riva I, Köhler J, Lötters S, Reichle S. 2000. Ten years of research on Bolivian amphibians: updated checklist, distribution, taxonomic problems, literature and iconography. Revista Española de Herpetología. 14:19-164.

Edgar RC. 2004. MUSCLE: multiple sequence alignment with high accuracy and high throughput. Nucleic Acids Research. 32:1792-1797.

Elith J, Graham CH, Anderson RP, Dudik M, Ferrier S, Guisan A, Hijmans RJ, Huettmann F, Leathwick JR, Lehmann A, et al. 2006. Novel methods improve prediction of species' distributions from occurrence data. Ecography. 29:129-151.

Ficetola GF, Rondinini C, Bonardi A, Katariya V, Padoa-Schioppa E, Angulo A. 2014. An evaluation of the robustness of global amphibian range maps. Journal of Biogeography. 41:211-221.

Ficetola GF, Thuiller W, Miaud C. 2007. Prediction and validation of the potential global distribution of a problematic alien invasive species - the American bullfrog. Diversity and Distributions. $13: 476-485$.

Fielding AH, Bell JF. 1997. A review of methods for the assessment of prediction errors in conservation presence/absence models. Environmental Conservation. 24:38-49.

Fouquet A, Gilles A, Vences M, Marty C, Blanc M, Gemmell NJ. 2007. Underestimation of species richness in Neotropical frogs revealed by mtDNA analyses. PLoS ONE. 2:e1109. doi: 10.1371/journal.pone.0001109 
Fouquet A, Loebmann D, Castroviejo-Fisher S, Padial JM, Orrico VGD, Lyra ML, Roberto IJ, Kok PJR, Haddad CFB, Rodrigues MT. 2012. From Amazonia to the Atlantic forest: molecular phylogeny of Phyzelaphryninae frogs reveals unexpected diversity and a striking biogeographic pattern emphasizing conservation challenges. Molecular Phylogenetics and Evolution. 65:547-561.

Frost DR. 2016. Amphibian species of the world: an online reference [Internet]. New York (NY): American Museum of Natural History. Version 6.0 [cited 2014 May 29]. Available from: http:// research.amnh.org/vz/herpetology/amphibia/.

Giovanelli JGR, Araújo CO, Haddad CFB, Alexandrino J. 2008b. Modelagem do nicho ecológico de Phyllomedusa ayeaye (Anura: Hylidae): previsão de novas áreas de ocorrência para uma espécie rara. Neotropical Biology and Conservation. 3:59-65.

Giovanelli JGR, Haddad CFB, Alexandrino J. 2008a. Predicting the potential distribution of the alien invasive American bullfrog (Lithobates catesbeianus) in Brazil. Biological Invasions. 10:585590.

Giovanelli JGR, de Siqueira MF, Haddad CFB, Alexandrino J. 2010. Modeling a spatially restricted distribution in the Neotropics: how the size of calibration area affects the performance of five presence-only methods. Ecological Modelling. 221:215-224.

Gordo M, Campos Z. 2003. Listagem de anuros da Estação Ecológica Nhumirim e arredores, Pantanal Sul. Documentos - Embrapa Pantanal, Corumbá, MS. 58:1-21.

Guisan A, Thuiller W. 2005. Predicting species distribution: offering more than simple habitat models. Ecology Letters. 8:993-1009.

Hijmans R, Cameron SE, Parra JL, Jones PG, Jarvis A. 2005. Very high resolution interpolated climate surfaces for global land areas. International Journal of Climatology. 25:1965-1978.

Hugall A, Moritz C, Moussalli A, Stanisic J. 2002. Reconciling paleodistribution models and comparative phylogeography in the wet tropics rainforest land snail Gnarosophia bellendenkerensis (Brazier 1875). Proceedings of the National Academy of Sciences. 99(9):6112-6117.

IUCN. 2016. The IUCN Red List of Threatened Species [Internet]. Version 2015.4 [cited 2016 July 16]. Available from: http://www.iucnredlist.org

Jansen M, Bloch R, Schulze A, Pfenninger M. 2011. Integrative inventory of Bolivia's lowland anurans reveals hidden diversity. Zoologica Scripta. 40:567-583.

Jeganathan P, Green RE, Norris K, Vogiatzakis IN, Bartsch A, Wotton SR, Bowden CGR, Griffiths GH, Pain D, Rahmani AR. 2004. Modelling habitat selection and distribution of the critically endangered Jerdon's courser Rhinoptilus bitorquatus in scrub jungle: an application of a new tracking method. Journal of Applied Ecology. 41:224-237.

Kokubum MNC, Menin M. 2002. Physalaemus centralis, Brazil. Herpetological Review. 33(1):62.

Lobo F. 1993. Descripción de una nueva especie del género Physalaemus (Anura: Leptodactylidae) del noroeste de la Argentina. Revista Española de Herpetología. 7:13-20.

Loebmann D, Haddad CFB. 2010. Amphibians and reptiles from a highly diverse area of the Caatinga domain: composition and conservation implications. Biota Neotropica. 10:227-256.

Lourenço LB, Targueta CP, Baldo D, Nascimento J, Garcia PCA, Andrade GV, Haddad CFB, Recco-Pimentel SM. 2015. Phylogeny of frogs from the genus Physalaemus (Anura, Leptodactylidae) inferred from mitochondrial and nuclear gene sequences. Molecular Phylogenetics and Evolution. 92:204-216.

Lyra ML, Haddad CFB, Azeredo-Espin AML. Meeting the challenge of DNA barcoding Neotropical amphibians: polymerase chain reaction optimization and new COI primers. Molecular Ecology Resources. 2016. doi:1111/1755-0998.12648.

Magalhães FM, Loebmann D, Kokubum MNC, Haddad CFB, Garda AA. 2014. A new species of Pseudopaludicola (Anura: Leptodactylidae: Leiuperinae) from Northeastern Brazil. Herpetologica. 70:77-88.

Nascimento LB, Caramaschi U, Cruz CAG. 2005. Taxonomic review of the species groups of the genus Physalaemus Fitzinger, 1826. Arquivos do Museu Nacional. 63:297-320.

Padial JM, Köhler J. 2001. First record of Physalaemus centralis (Anura: Leptodactylidae) for Bolivia, with description of its advertisement call. Boletín de la Asociación Herpetológica Española. 12:6-8.

Papes M, Peterson AT. 2003. Predicting the potential invasive distribution for Eupatorium adenophorum Spreng in China. Journal of Wuhan Botanical Research. 21:137-142.

Peloso PLV, Sturaro MJ, Forlani MC, Gaucher P, Motta AP, Wheeler WC. 2014. Phylogeny, taxonomic revision, and character evolution of the genera Chiasmocleis and Syncope (Anura, Microhylidae) in Amazonia, with descriptions of three new species. Bulletin of the American Museum of Natural History. 386:1-96, 15 pls. 
Peterson AT, Scachetti-Pereira R, Kluza DA. 2003. Assessment of invasive potential of Homalodisca coagulata in western North America and South America. Biota Neotropica [Internet]. Available from: http://www.biotaneotropica.org.br/v3n1/pt/abstract?article+BN00703012003

Phillips SJ, Anderson RP, Schapire RE. 2006. Maximum entropy modeling of species geographic distributions. Ecological Modelling. 190:231-259.

Prado VHM, Da Silva FR, Dias NYN, Pires JSR, Rossa-Feres DC. 2009. Anura, Estação Ecológica de Jataí, São Paulo state, southeastern Brazil. Check List. 5:495-502.

Prado CPA, Haddad CFB. 2005. Size-fecundity relationships and reproductive investment in female frogs in the Pantanal, south-western Brazil. Herpetological Journal. 15:181-189.

Ratnasingham S, Hebert PDN. 2007. BARCODING: bold: the barcode of life data system (http:// www.barcodinglife.org). Molecular Ecology Notes. 7:355-364.

Roberto IJ, Loebmann D. 2016. Composition, patterns of distribution and conservation priority areas for the herpetofauna in the state of Ceará, northeastern Brazil. Salamandra. 52:134-152.

Roberto IJ, Ribeiro SC, Loebmann D. 2013. Amphibians of the state of Piauí, Northeastern Brazil: a preliminary assessment. Biota Neotropica. 13:322-330.

Silveira AL. 2006. Anfíbios do município de João Pinheiro, uma área de cerrado no noroeste de Minas Gerais, Brasil. Arquivos do Museu Nacional. 64:131-139.

Smith P, Cacciali P, Atkinson K, Pheasey H, Motte M. 2012. New distributional records of amphibians for Departamento San Pedro, Paraguay (Amphibia). Check List. 8:903-907.

Strüssmann C, Prado CPA, Uetanabaro M, Ferreira VL. 2000. Amphibian and reptile survey of selected localities in the southern Pantanal floodplains and surrounding Cerrado, Mato Grosso do Sul, Brasil. In: Willink PW, Chernoff B, Alonso LE, Montambault JR, Lourival R, org. A biological assessment of the aquatic ecosystems of the Pantanal, Mato Grosso do Sul, Brasil. Vol. 18, 1st ed. Washington, DC: Conservation International. p. 98-102.

Tamura K, Stecher G, Peterson D, Filipski A, Kumar S. 2013. MEGA6: molecular evolutionary genetics analysis version 6.0. Molecular Biology and Evolution. 30:2725-2729.

Teixeira Jr. M, Vechio FD, Recoder RS, Carnaval AC, Strangas M, Damasceno RP, Sena MA, Rodrigues MT. 2012. Two new species of marsupial tree-frogs genus Gastrotheca Fitzinger, 1843 (Anura, Hemiphractidae) from the Brazilian Atlantic Forest. Zootaxa. 3437:1-23.

Toledo LF, Zina J, Haddad CFB. 2003. Distribuição espacial e temporal de uma comunidade de anfíbios anuros do município de Rio Claro São Paulo. Holos Environment. 3:136-149.

Tombini AAM, Béda AF. 2002. Dados Preliminares do Levantamento Herpetológico da área de influência do Gasoduto Campo Grande Dourados- MS. In: I Encontro Científico da Universidade Estadual de Mato Grosso do Sul, Vol. 1. Dourados, MS: Editora da UEMS. p. 57.

Valdujo PH, Recoder RS, Vasconcelos MM, Portella AS. 2009. Amphibia, Anura, São Desidério, western Bahia uplands, northeastern Brazil. Check List. 5:903-911.

Van-Silva W, Guedes AG, Azevedo-Silva PL, Gontijo FF, Barbosa RS, Aloísio GR, Oliveira FCG. 2007. Herpetofauna, Espora Hydroelectric Power Plant, state of Goiás, Brazil. Check List. 3(4):338-345.

Vasconcelos TS, Rossa-Feres DC. 2005. Diversidade, distribuição espacial e temporal de anfíbios anuros (Amphibia, Anura) na região noroeste do estado de São Paulo, Brasil. Biota Neotropica. $5: 1-14$.

Vrcibradic D, Almeida-Gomes M, Van Sluys MV, Rocha CFD. 2008. Amphibia, Anura, Hylodes charadranaetes, Ischnocnema octavioi, and Euparkerella cochranae: distribution extension. Check List. 4:103-106.

Werneck FP. 2011. The diversification of eastern South American open vegetation biomes: historical biogeography and perspectives. Quaternary Science Reviews. 30:1630-1648.

Young BE, editor. 2007. Distribución de las especies endémicas en la vertiente oriental de los Andes en Perú y Bolivia. Arlington, TX: NatureServe. 89 p. 


\section{Appendix 1.}

Specimens of Physalaemus cuvieri group morphologically analysed.

Physalaemus centralis. BRAZIL. State of Goiás, municipality of Baliza (CFBH 688), state of Bahia, municipality of Lençóis (CFBH 6659, 6660), municipality of São Desidério (CFBH 20539), municipality of Jaborandi (CFBH 20551), municipality of Bahianópolis (CFBH 21007), municipality of Caetité (CFBH 21079, 21080); state of Minas Gerais, municipality of Belo Horizonte (CFBH 1479, 1480); state of Mato Grosso do Sul, municipality of Três Lagoas (CFBH 13595, 18482, 18483, 22631, 24016, 24020, 24040); state of Mato Grosso, municipality of Chapada dos Guimarães (CFBH 14392); state of Piauí, municipality of Piracuruca (CFBH 14392, 19396); state of Rondônia, municipality of Colorado do Oeste (CFBH 20424); state of São Paulo, municipality of Corumbataí (CFBH 1340, 2057), municipality of Rio Claro (CFBH 2368, 4580), municipality of Itirapina (CFBH 5830), municipality of Brotas (CFBH 9805, 9830, 9831), municipality of Angatuba (CFBH 23118, 23138, 23149); state of Tocantins, municipality of Caseara (CFBH 10282), municipality of Araguacema (CFBH 10313), municipality of Wanderlândia (CFBH 11452), municipality of Lizarda (CFBH 13221), municipality of Gurupi (CFBH 20962, 20963, 22035).

Physalaemus albifrons (Spix, 1824): BRAZIL. State of Ceará, municipality of Caucaia (UFCA 4093), municipality of Jati (MNRJ 55529), municipality of Caucaia (UFCA 4093), São Gonçalo do Amarante (URCA-H 5603, 6401, 6405), municipality of Trairi (URCA-H 5666), municipality of Viçosa do Ceará (CFBH 16137-16141).

Physalaemus albonotatus (Steindachner, 1864): BRAZIL. State of Mato Grosso, municipality of Cáceres, ZUEC 7067-7-78 (topotypes), municipality of Acorizal, CFBH 14240, 14142-14144, municipality of Dom Aquino, CFBH14331-14332; State of Mato Grosso do Sul, municipality of Bonito, CFBH 14253-14254; municipality of Corumbá, CFBH 8987; state of Tocantins, municipality of Mateiros, MZUSP 133262-133267.

Physalaemus cuvieri Fitzinger, 1826: BRAZIL. State of Tocantins, municipality of Babaçulândia, CFBH 11408; municipality of Araguacema, CFBH 10291; state of São Paulo, municipality of Assis, CFBH 20015; municipality of Itirapina, CFBH 5712, 6026, 9828, 9838, 21934; municipality of Mauá, CFBH 9908; municipality of São Paulo, CFBH 9140; municipality of São José do Rio Preto, CFBH 9139; CFBH 11316. municipality of Rio Claro, CFBH 5587; state os Santa Catarina, municipality of Campos Novos, CFBH 13639.

Physalaemus ephippifer (Steidachner, 1864): BRAZIL. State of Pará, municipality of Belém, MNRJ 32276-80, MNRJ 69637-69644 (topotypes); ZUEC 13724-13741.

Physalaemus erikae Cruz and Pimenta, 2004: BRAZIL. State of Bahia, minicipality of Graratinga, MNRJ 35401 (topotype); municipality of Ilhéus, MNRJ 48647-50.

Physalaemus fischeri (Boulenger, 1890): VENEZUELA. Province of Apure, municipality of Mantecal, ZUEC 10475-10476, 9369, 9362.

Physalaemus kroyeri (Reinhardt and Lutken, 1862): BRAZIL. State of Bahia, municipality of Maracás, MZUSP 96472-96547. 


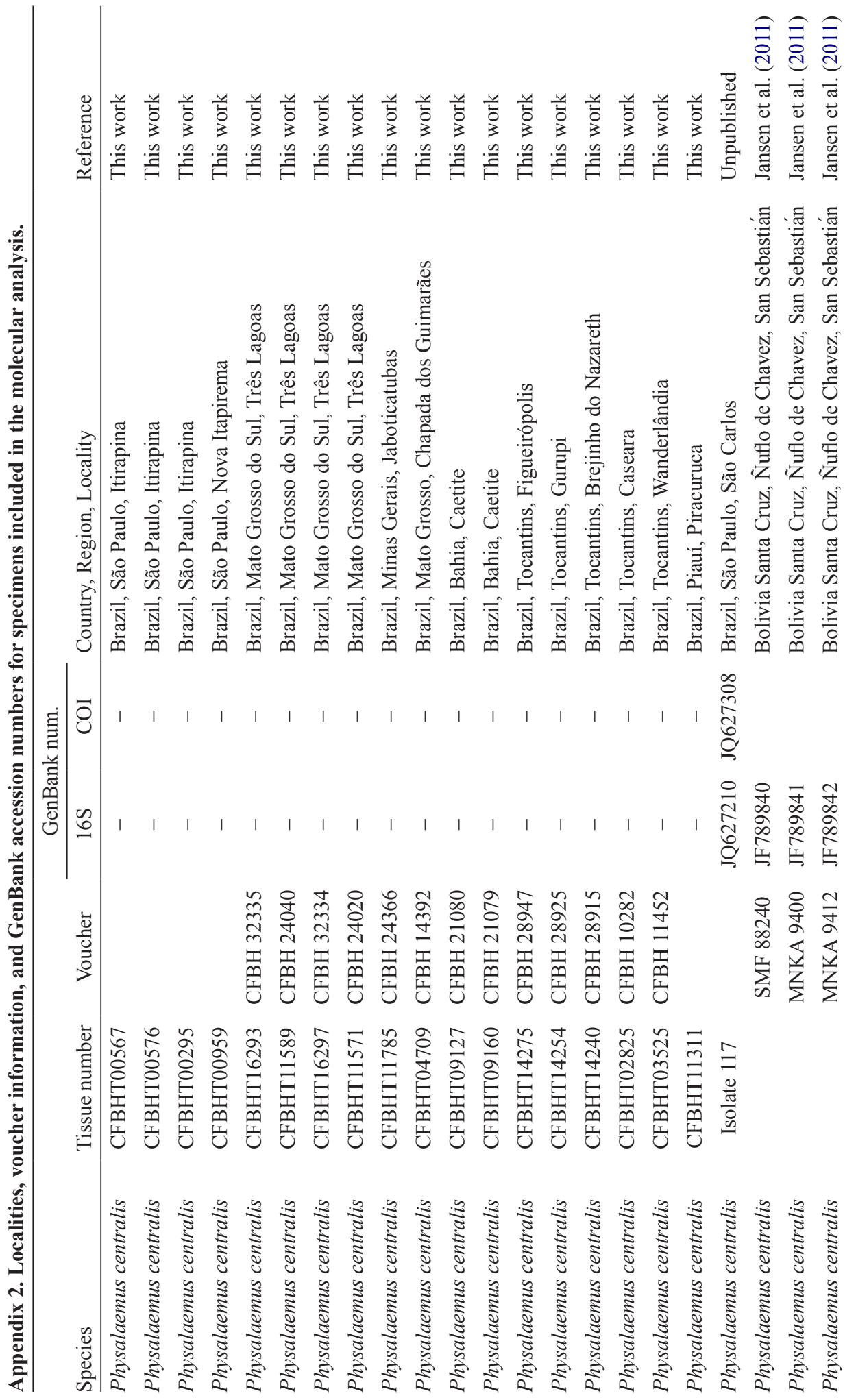




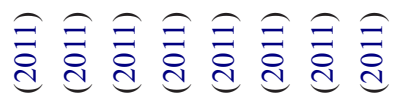

ब

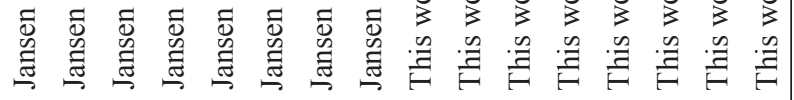

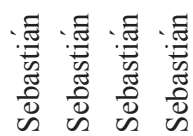

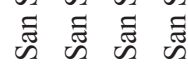

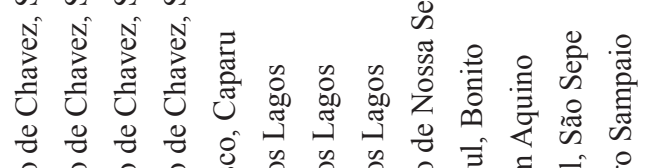

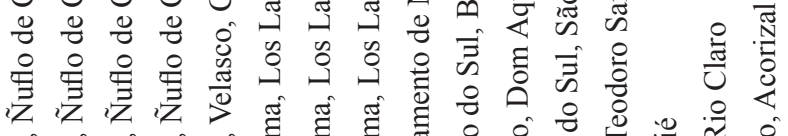

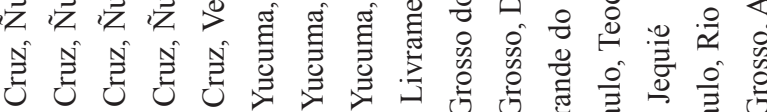

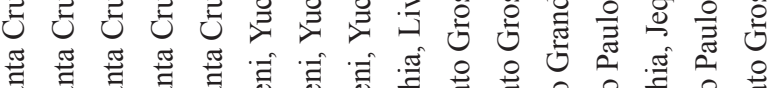

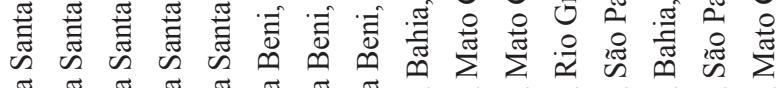

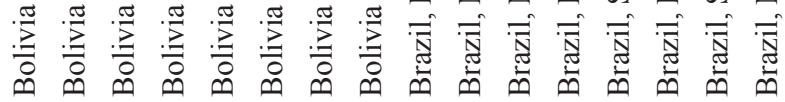

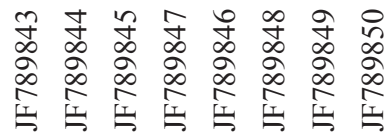

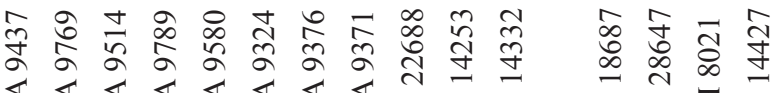

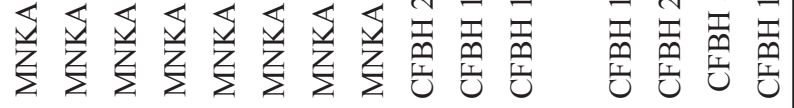

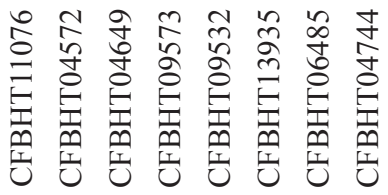

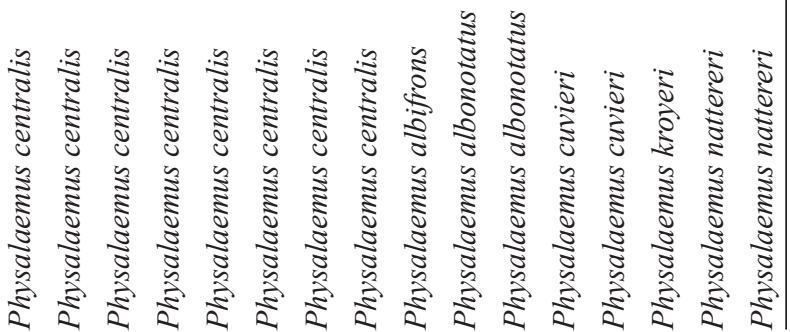

